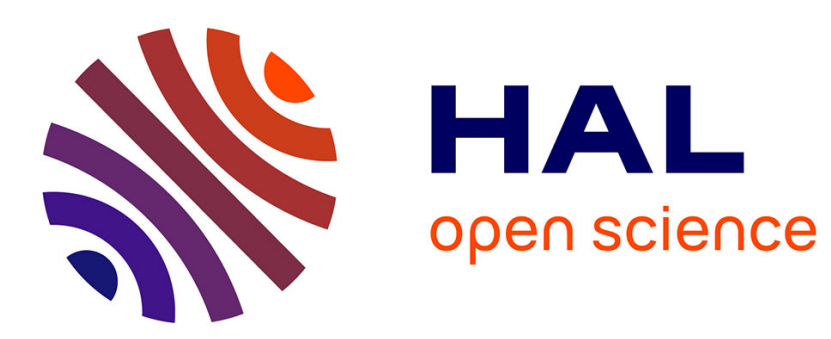

\title{
Complexity gain of QR Decomposition based Sphere Decoder in LTE receivers
}

Sébastien Aubert, Fabienne Nouvel, Amor Nafkha

\section{To cite this version:}

Sébastien Aubert, Fabienne Nouvel, Amor Nafkha. Complexity gain of QR Decomposition based Sphere Decoder in LTE receivers. VTC Fall 2009, Sep 2009, Anchorage, United States. 6 p. hal00444383

\section{HAL Id: hal-00444383 \\ https://hal.science/hal-00444383}

Submitted on 6 Jan 2010

HAL is a multi-disciplinary open access archive for the deposit and dissemination of scientific research documents, whether they are published or not. The documents may come from teaching and research institutions in France or abroad, or from public or private research centers.
L'archive ouverte pluridisciplinaire HAL, est destinée au dépôt et à la diffusion de documents scientifiques de niveau recherche, publiés ou non, émanant des établissements d'enseignement et de recherche français ou étrangers, des laboratoires publics ou privés. 


\section{Complexity gain of QR Decomposition based Sphere Decoder in LTE receivers}

\author{
Sébastien Aubert ${ }^{\dagger *}$ \\ $\dagger$ ST-NXP Wireless; \\ 505, route des lucioles; \\ CP 06560 Sophia-Antipolis France \\ Email: sebastien.aubert@stnwireless.com Email: fabienne.nouvel@insa-rennes.fr \\ Fabienne Nouvel ${ }^{\dagger}$ \\ $\dagger$ Research unit IETR; \\ 20, avenue des buttes de Coësmes; \\ CP 35043 Rennes France
}

\begin{abstract}
It has been widely shown that the Sphere Decoding can be used to find the Maximum Likelihood (ML) solution with an expected complexity that is roughly cubic in the dimensions of the problem. However, the computational complexity becomes prohibitive if the Signal-to-Noise Ratio is too low and/or if the dimension of the problem is too large. That is why another technique denoted as Fixed-complexity Sphere Decoder (FSD) is an interesting approach. This algorithm needs a preprocessing step, and in this paper the QR-Decomposition-based preprocessing technique, which is not inconsequential, will be studied. Two different techniques are exposed, including the classical Gram Schmidt orthonormalization process. Their computational complexities and their impacts on the FSD computational complexity are studied. In the LTE context, the overall computational complexities of the two detection techniques are quantified and are shown to be dependent on the constellation size.

Index Terms-MIMO detection, Sphere Decoder, QR Decomposition, Householder, LTE.
\end{abstract}

\section{INTRODUCTION AND PROBLEM STATEMENT}

One of the main challenges in receiver design for wireless communication systems lies in the non-orthogonality of the transmission channel which implies a performance degradation of the system due to Inter-Layer Interferences (ILI). So, to ensure high reliability of data transmission, a special attention has to be paid to the receiver block. The system model, made of $n_{T}$ and $n_{R}$ transmit and receive antennas respectively, reads

$$
\mathbf{y}=\mathbf{H x}+\mathbf{n},
$$

where $\mathbf{x} \in \mathbb{C}^{n_{T}}$ and $\mathbf{y} \in \mathbb{C}^{n_{R}}$ denote the system input and output respectively, the matrix $\mathbf{H} \in \mathbb{C}^{n_{R} \times n_{T}}$ representing the equivalent channel matrix, and $\mathbf{n} \in \mathbb{C}^{n_{R}}$ the additive white gaussian noise. Typically, the noise components are independent and identically distributed as zero-mean Gaussian random variables with a variance $\sigma^{2}$. The entries of $\mathbf{x}$ are assumed to be uniformly distributed over a finite set of symbols $\xi^{n_{T}}$. The channel matrix $\mathbf{H}$ is assumed to be perfectly known at the receiver end.

The optimum Maximum Likelihood (ML) detection [1] consists in finding the signal vector $\hat{\mathbf{x}} \in \xi^{n_{T}}$ that minimizes the Euclidean distance with respect to the received signal vector y. $\hat{\mathbf{x}}$ is the closest lattice point [2] in the Euclidean space

$$
\hat{\mathbf{x}}=\underset{\mathbf{x} \in \xi^{n} T}{\operatorname{argmin}}\|\mathbf{y}-\mathbf{H x}\|_{2}^{2} .
$$

An exhaustive search over all feasible elements $\xi^{n_{T}}$ is necessary to solve ML problems (2). However, the computational complexity is exponential in the number of possible constellation points, making this technique unsuitable for practical purposes when aiming at high modulation orders and numbers of transmit antennas. Nevertheless, for low dimension problems with low modulation order schemes such as BPSK and QPSK, exhaustive search methods have been shown to be feasible [3]. For higher problem dimension, the ML detection problem (2) can be resolved with a reasonable complexity using an efficient search method like the universal lattice decoding algorithm [4], also called Sphere Decoder (SD). The SD search algorithm is based on the Finke-Pohst enumeration to explore all the lattice points inside a hypersphere centered at the received symbol vector [5]. The interest in lattice decoding has steadily grown in the last few years. One of the most efficient known SD algorithms has been proposed by Viterbo and Boutros (VB) in [6]. However, one important drawback in the VB algorithm and other similar SD algorithms is the choice of the initial value of the search radius. If this radius is chosen too small, there may be no solution for the algorithm (no point inside the hypersphere) and if the radius is chosen too large, the number of explored points may be very high and the algorithm will be inefficient, acting like the ML algorithm. Although there are some methods for deciding on the initial value of the radius, there is not yet any automatic method that works well in all different applications. In all proposed methods, it is still possible that no valid point is found inside the hypersphere. In those cases, the search procedure must be repeated with a larger radius. This reiteration considerably increases the computation time. In some cases, like signal detection in fading channels, the choice of this radius is even more difficult [6]: because of the rapid changes in the channel condition, the initial value of this radius must be determined more frequently.

The average complexity of the SD algorithm is shown to be polynomial (roughly cubic) in the number of transmit antennas over a certain range of Signal-to-Noise Ratio (SNR) and number of transmit antennas, while the worst case complexity is still exponential [7].

From a hardware implementation point of view, the SD algorithm exhibits two major weaknesses. First, its complexity 
coefficients can become large when the problem dimension is high, i.e. at the spectral efficiency demanded by future devices. Second, the variance of its computation time can also be large, leading to undesirable highly variable decoding delays.

For the sake of lower computational complexity and simplicity for the hardware implementation, the use of the Fixedcomplexity SD (FSD) is interesting. This detector has been previously proposed for the detection in uncoded MIMO systems using Quadrature Amplitude Modulation (QAM) constellations [8]. It overcomes the two main drawbacks of the $\mathrm{SD}$ from an implementation point of view, i.e. its variable complexity depending on the noise level, and the sequential nature of its tree search phase.

The next Section briefly reviews the state of the art in the existing FSD algorithm. Subsequently, in Section III, the contribution is exposed with the description of two efficient preprocessing methods through their computational complexity and stability [9]. To demonstrate the efficiency of our approach to reduce the computational complexity of the FSD, several simulation results and comparative analysis are provided in Section IV. The paper concludes with a summary in Section $\mathrm{V}$.

\section{FiXed-COMPlexity Sphere Decoder}

The FSD was introduced in [8], [10] to overcome some of the problems faced by the original SD in relation to its hardware implementation. Specifically, it addressed the variable complexity of the SD and the sequentiality of certain parts of the SD algorithm which made a pipelined implementation difficult. The FSD can in essence be described as a partial search through a small subset of the constellation using a specific detection ordering. In [8], it was shown that the FSD achieves maximal diversity in reception when applied to the two by two independent and identically distributed Rayleigh fading multiple antenna channel.

The FSD performs a search over a fixed number of lattice points $\mathbf{H x}$, generated by a small subset $S$ included in $\xi^{n_{T}}$, around the received vector $\mathbf{y}$. The transmitted vector $\mathbf{x}$ which belongs to $S$ associated to the smallest Euclidean distance is then selected as the estimate (2).

The processing and preprocessing parts will be presented in the following Subsections.

\section{A. Preprocessing of FSD}

The channel matrix $\mathbf{H}$ is first decomposed using a $\mathbf{Q R}$ Decomposition (QRD) method. Hence, $\mathbf{H}=\mathbf{Q R}$, where $\mathbf{Q}$ is a $n_{R} \times n_{T}$ unitary matrix, i.e. $\mathbf{Q}^{H} \mathbf{Q}=\mathbf{I}$, and $\mathbf{R}$ is a $n_{T} \times n_{T}$ upper triangular matrix. Then, the received symbol vector $\mathbf{y}$ is multiplied with $\mathbf{Q}^{H}$ prior to the symbol detection step, and (1) reads

$$
\mathbf{Q}^{H} \mathbf{y}=\mathbf{Q}^{H} \mathbf{Q R} \mathbf{x}+\mathbf{Q}^{H} \mathbf{n}=\mathbf{R} \mathbf{x}+\mathbf{Q}^{H} \mathbf{n} .
$$

The matrix $\mathbf{R}$ structure being upper triangular, the transmitted symbol vector $\mathbf{x}$ could be estimated from the vector $\mathbf{Q}^{H} \mathbf{y}$ denoted as $\tilde{\mathbf{y}}$ and the matrix $\mathbf{R}$ by employing the Gauss elimination algorithm [9] or the proposed Householder algorithm (see Section III). Denoting $\mathbf{Q}^{H} \mathbf{n}$ as $\tilde{\mathbf{n}}$, (3) reads

$$
\tilde{\mathbf{y}}=\mathbf{R x}+\tilde{\mathbf{n}} .
$$

Note that $\mathbf{Q}$ being an unitary matrix, the covariance of the noise term remains unaffected.

Assuming the QRD is realized and due to the shape of $\mathbf{R},(1)$ can be written in an iterative monotonically increasing form

$$
\hat{\mathbf{x}}=\underset{\mathbf{x} \in \xi^{n_{T}}}{\operatorname{argmin}}\|\tilde{\mathbf{y}}-\mathbf{R} \mathbf{x}\|_{2}^{2}=\underset{\mathbf{x} \in \xi^{n_{T}}}{\operatorname{argmin}} \sum_{i=1}^{n_{T}} \delta_{i} \leq C
$$

where $C$ is the sphere constraint and $\delta_{i}$ denotes the Partial Euclidean Distance (PED) corresponding to the symbol transmitted on the $i$-th layer and such as

$$
\delta_{i}=\left\|\tilde{y}_{i}-\sum_{j=i}^{n_{T}} r_{i j} x_{j}\right\|_{2}^{2}
$$

for all $1 \leq i \leq n_{T}$.

\section{B. Processing of FSD}

Thanks to the PED introduction, the optimal symbols vector search can be depicted as a tree search [11], with the first node corresponding to $n_{T}$-th symbol at the top level.

Figure 1 illustrates the tree construction for two transmit antennas. The FSD algorithm idea is to apply a full ML search at the top layer and a linear equalizer on the rest of the layers. Concerning the tree exploration, whether the sphere constraint is respected or not, the following explored symbol vector is tested by stepping down the tree from the root node, according to the depth-first search principle. The Reference FSD (RFSD) algorithm is detailed in [12].

The basic principle induces some optimizations such as Schnorr-Euchner (SE) enumeration which was introduced in [13], [2]. It was shown in [14] that enumerating the symbols at the top-level of the tree in an ascending order according to their distance to the Babai point [2] will expedite the tree search, as depicted in Figure 1. Thus, the processing

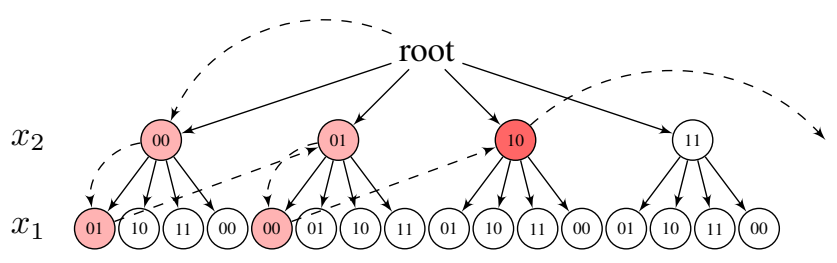

$\bigcirc$ Node not processed

- Node processed with $\delta_{1}<C$

- Node processed with $\delta_{2} \geq C$

Fig. 1. Tree representation of FSD.

complexity is decreased by reducing the number of visited nodes. In addition, the problematic setting of a proper initial radius becomes useless: once the Babai point is obtained, the radius is shrunk to the total computed Euclidean distance. The processing complexity can also be decreased through 
the preprocessing step, which is presented in the following Subsections. The preprocessing algorithm stability [9] point of view is also an important aspect since the loss of orthogonality in the matrix $\mathbf{Q}$ (due to the round-off error) induces ILI and reduces the FSD performance.

In this paper, Gram-Schmidt (GS) orthogonalization and Householder $(\mathrm{HH})$ transformation are described as well as their computational complexities and their impact on the processing computational complexity.

\section{ENHANCED PREPRocessing SteP}

\section{A. Gram-Schmidt}

The GS algorithm [9] is an orthogonalization procedure to get an orthonormal basis $\mathbf{Q}=\left(\mathbf{q}_{1}, \mathbf{q}_{2}\right)$ from any invertible matrix $\mathbf{H}=\left(\mathbf{h}_{1}, \mathbf{h}_{2}\right)$, as described in Figure 2 which geometrically illustrates the first two steps of the orthogonalization procedure. The $\left(\mathbf{h}_{1}, \mathbf{h}_{2}\right)$ basis is transformed

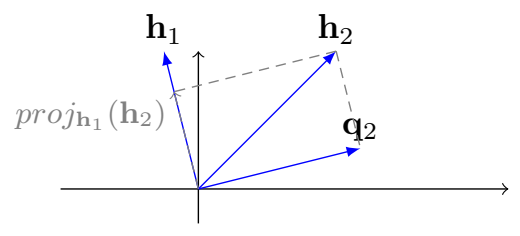

Fig. 2. GS orthogonalization principle.

into the orthonormal $\left(\mathbf{q}_{1}, \mathbf{q}_{2}\right)$ basis thanks to the orthogonal projection of $\mathbf{h}_{2}$ onto the subspace generated by $\mathbf{q}_{1}$ (which is the same as the subspace generated by $\mathbf{h}_{1}$ ). $\mathbf{q}_{2}$ is then defined as $\mathbf{h}_{2}-\operatorname{proj}_{\mathbf{h}_{1}}\left(\mathbf{h}_{2}\right)$, with $\operatorname{proj}_{\mathbf{u}}(\mathbf{v})$ the projection of $\mathbf{v}$ on $\mathbf{u}$ such that $\operatorname{proj}_{\mathbf{u}}(\mathbf{v})=\frac{\mathbf{u}^{H} \mathbf{v}}{\mathbf{u}^{H} \mathbf{u}} \mathbf{u}$. The obtained set of orthogonal vectors finally has to be normalized, so that a set of orthonormal vectors is obtained.

With this technique, the matrix $\mathbf{Q}$ is determined first, then the matrix $\mathbf{R}$ is deduced using $\mathbf{Q}$ and $\mathbf{H}$. It can be shown that $\mathbf{R}=\left(\begin{array}{cc}r_{11} & r_{12} \\ 0 & r_{22}\end{array}\right)$, with $r_{11} \in \mathbb{R}_{+}$and $r_{12}, r_{22} \in \mathbb{C}$.

Another technique, which results in real positive coefficients rather than complex ones, may be used.

\section{B. Householder}

The $\mathrm{HH}$ algorithm [9] is a transformation procedure to get an upper triangular matrix $\mathbf{R}$, as described in Figure 3 which geometrically illustrates the first two steps of the reflection procedure. A Householder matrix, which is also

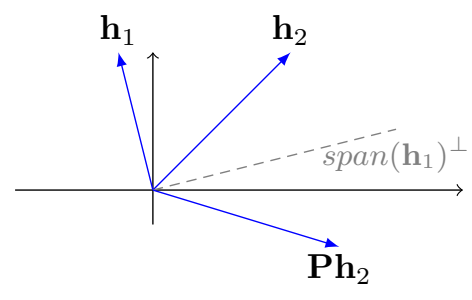

Fig. 3. HH transformation principle.

known as a Householder reflector, is a matrix of the form
$\mathbf{P}_{\mathbf{v}}=\mathbf{I}-2 \frac{\mathbf{v v}^{H}}{\mathbf{v}^{H} \mathbf{v}}$, with $\mathbf{v} \in \mathbb{C}^{2 *}$. The application of $\mathbf{P}_{\mathbf{v}}$ to a vector $\mathbf{x}$ yields $\mathbf{P}_{\mathbf{v}} \mathbf{x}=\mathbf{x}-2 \frac{\mathbf{v}^{H} \mathbf{x}}{\mathbf{v}^{H} \mathbf{v}} \mathbf{v}=\mathbf{x}-2 \cdot \operatorname{proj}_{\mathbf{v}}(\mathbf{x})$.

Figure 3 illustrates the reflection of $\mathbf{h}_{2}$ with respect to the hyperplane $\operatorname{span}\left(\mathbf{h}_{1}\right)^{\perp}$. Householder matrices are a mathematical tool for introducing zeros into vectors, such as any matrix can be made upper triangular with an iterative processing, without need of computing explicitly the Householder matrix.

With this technique, the matrix $\mathbf{R}$ is determined first, then the matrix $\mathbf{Q}$ is deduced using $\mathbf{R}$ and $\mathbf{H}$. It can be shown that $\mathbf{R}=\left(\begin{array}{cc}r_{11} & r_{12} \\ 0 & r_{22}\end{array}\right)$, with $r_{11}, r_{22} \in \mathbb{R}_{+}$and $r_{12} \in \mathbb{C}$.

The detector, associated with the HH-based preprocessing, which takes into account the occurrence of a real positive coefficient in the matrix $\mathbf{R}$ is denoted as the Enhanced FSD (EFSD). Even if the $\mathrm{HH}$ algorithm implies a fixed increase complexity of the QRD, the EFSD complexity is largely decreased compared to the RFSD. In Section IV, the overall complexity changes will be quantified.

\section{Householder and Gram Schmidt numerical stability}

It has been shown in [9] that the GS-based QRD computational complexity is lower than the HH-based QRD's. In particular, the GS method produces $\mathrm{Q}$ explicitly unlike the $\mathrm{HH}$ method which holds the matrix $\mathrm{Q}$ in factored form.

Nevertheless, it is shown in Figure 4 that the GS-based QRD produces a loss of orthogonality of the matrix $\mathbf{Q}$ $\left(\left\|\mathbf{Q}^{H} \mathbf{Q}-\mathbf{I}\right\|_{\mathrm{F}} \neq 0\right.$, where $\|\cdot\|_{\mathrm{F}}$ denotes the Frobenius norm), especially in ill-conditioned channel matrix case, commonly observed in LTE systems because of the presence of correlation between antennas.

The ratio

$$
\operatorname{Cond}(\mathbf{H})=\frac{\left|\lambda_{\max }\right|}{\left|\lambda_{\min }\right|}
$$

is called condition number, with $\lambda_{\max }$ and $\lambda_{\min }$ respectively denoting maximal and minimal eigenvalues of $\mathbf{H}$. It measures the degree of singularity of $\mathbf{H}$. In particular, when $\operatorname{Cond}(\mathbf{H})$ is large, the matrix $\mathbf{H}$ is ill-conditioned and when $\operatorname{Cond}(\mathbf{H})$ is very small (close to 1 ) the matrix $\mathbf{H}$ becomes more orthogonal. In Figure 4, the ratio of the loss of orthogonality with the GS technique by the loss of orthogonality with the $\mathrm{HH}$ technique is depicted versus the condition number, in a complex $5 \times 5$ matrix case. Thus, the $\mathrm{HH}$ tehnique is more stable than the stable version GS technique [9], even in the case of a QRD of a quasi-orthogonal matrix.

In the sequel, the two preprocessing results are developed and compared, in particular in terms of computational complexities.

\section{Householder and Gram Schmidt complexities}

It can be shown that GS orthonormalization of
$\mathbf{H}=\left(\begin{array}{ll}h_{11} & h_{12} \\ h_{21} & h_{22}\end{array}\right)$ gives $\mathbf{H}=\mathbf{Q R}$ with

$$
\mathbf{Q}=\left(\begin{array}{ll}
\frac{h_{11}}{\left\|\mathbf{h}_{1}\right\|_{2}} & \frac{h_{21}^{*}}{\left\|\mathbf{h}_{1}\right\|_{2}} \\
\frac{h_{21}}{\left\|\mathbf{h}_{1}\right\|_{2}} & \frac{-h_{11}^{*}}{\left\|\mathbf{h}_{1}\right\|_{2}}
\end{array}\right)
$$




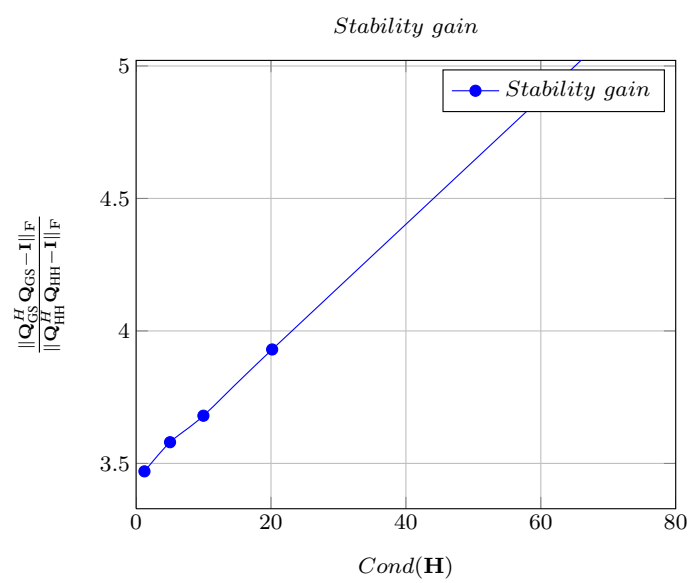

Fig. 4. Loss of orthogonality in the computed $\mathbf{Q}$ versus Condition Number.

$$
\mathbf{R}=\left(\begin{array}{cc}
\left\|\mathbf{h}_{1}\right\|_{2} & h_{11}^{*} h_{12}+h_{21}^{*} h_{22} \\
0 & h_{12} h_{21}-h_{11} h_{22}
\end{array}\right) .
$$

The $\mathrm{HH}$ reflection results are given below

$$
\begin{gathered}
\mathbf{Q}=\left(\begin{array}{cc}
\frac{h_{11}}{\left\|\mathbf{h}_{1}\right\|_{2}} & \frac{h_{21}^{\prime}}{\left\|\mathbf{h}_{2}^{\prime}\right\|_{2}} \\
\frac{h_{21}}{\left\|\mathbf{h}_{1}\right\|_{2}} & \frac{h_{22}^{\prime}}{\left\|\mathbf{h}_{2}^{\prime}\right\|_{2}}
\end{array}\right), \\
\mathbf{R}=\left(\begin{array}{cc}
\left\|\mathbf{h}_{1}\right\|_{2} & k\left\|\mathbf{h}_{1}\right\|_{2} \\
0 & \left\|\mathbf{h}_{\mathbf{2}}^{\prime}\right\|_{2}
\end{array}\right),
\end{gathered}
$$

with $k=\frac{\mathbf{h}_{1}^{H} \mathbf{h}_{2}}{\mathbf{h}_{1}^{H} \mathbf{h}_{1}}$ and $\mathbf{h}_{2}^{\prime}=\mathbf{h}_{2}-k \mathbf{h}_{1}$.

A complexity change due to HH-based QRD and to EFSD adaptation can be quantified, in order to come to a conclusion for the overall complexity evolution in LTE context.

In Table I, an approximation (real additions are ignored) of the computational complexity in terms of real multiplications, denoted as MUL, of the GS-based and the HH-based QRD are presented in a complex two by two MIMO case.

Thanks to the occurrence of a real positive coefficient in the

\begin{tabular}{cc}
\hline GS & HH \\
\hline 94 & 141 \\
\hline
\end{tabular}

TABLE I

QRD COMPLEXITIES.

matrix $\mathbf{R}$ diagonal, during the preprocessing step (instead of a complex coefficient), the processing complexity is impacted. More generally, the FSD computational complexity is variable and depends on the number of explored branches of the tree, called in this paper Paths Explorations (PE). A PE denotes the path from the root to a leaf of the tree (see Figure 1). In Table II, RFSD and EFSD computational complexities in terms of MUL are given for different PE numbers.

\section{Simulation Results}

In this Section, simulation results in terms of complexity and performance are shown.

\begin{tabular}{rrr}
\hline PE & RFSD & EFSD \\
\hline 2 & 68 & 38 \\
10 & 292 & 190 \\
40 & 1132 & 760 \\
\hline
\end{tabular}

TABLE II

FSD COMPLEXITIES.

\section{A. Fixed Complexity Sphere Detector complexity overview in LTE context}

The overall computational complexities in terms of MUL of the reference GS-based RFSD (GS-RFSD) and of the proposed HH-based EFSD (HH-EFSD), in different PE numbers cases, are shown in Table III.

The fixed computational complexity increase in preprocessing

\begin{tabular}{rrr}
\hline PE & GS-RFSD & HH-EFSD \\
\hline 2 & 162 & 187 \\
10 & 386 & 339 \\
40 & 1226 & 909 \\
\hline \multicolumn{3}{c}{ TABLE III } \\
QRD AND FSD COMPLEXITIES.
\end{tabular}

step and the variable computational complexity decrease in the detection step imply a switching point between the GS-RFSD and the HH-EFSD techniques. When less than 5 PE are performed, the computational complexity of the GS-RFSD is the lowest, whereas when more than 5 PE are performed, the computational complexity of the HH-EFSD is the lowest. In order to highlight this threshold, the ratio

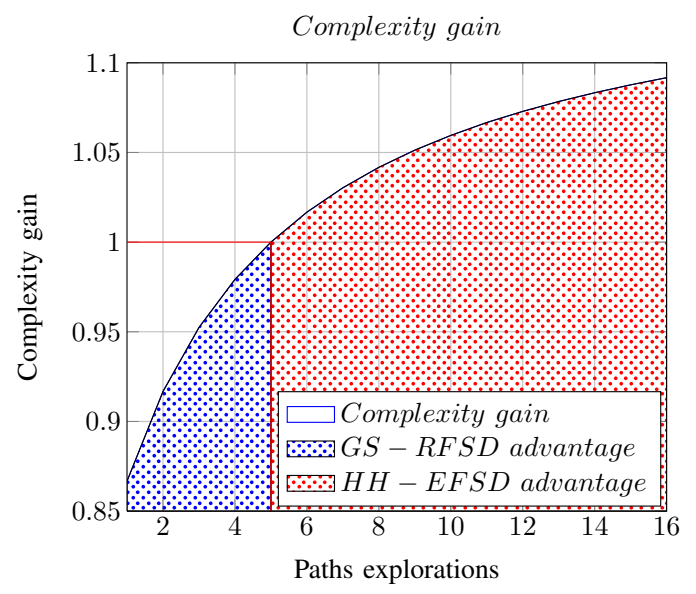

Fig. 5. Complexity gain between GS-RFSD and HH-EFSD versions.

of overall computational complexities of GS-RFSD by overall computational complexities of HH-EFSD is plotted in Figure 6 and shows that the overall computational complexity gain grows with the number of PE. The plot confirms that the proposed technique overall computational complexity is lower than the reference technique as soon as $5 \mathrm{PE}$ or more 
are needed. Therefore the proposed detector is more efficient in term of computational complexity for the $10 \mathrm{PE}$ calibration point, which is considered to be the medium PE number in LTE context [15].

\section{B. Fixed Complexity Sphere Detector performance overview in LTE context}

Considering the proposed detector, this solution performance is compared to the ML and the reference detector performances. The three techniques are plotted in Figure 7 without channel coding. The GS-RFSD and the HH-EFSD performances in terms of Bit Error Rate (BER) are superposed in order to check the equivalence of the two methods.

Simulation conditions of ML, GS-RFSD and HH-EFSD are given in Table IV. Figure 7 shows how curves are lay-

\begin{tabular}{ll}
\hline Parameter & Value \\
\hline Number of sub-carriers & 1 \\
Code rate & 1 \\
Mapping & LTE \\
\hline Channel simulation & MIMO $2 \times 2$ \\
Rayleigh \\
Channel estimation & Perfect \\
\hline Number of simulated channels & $10^{5}$ \\
\hline
\end{tabular}

TABLE IV

SIMULATION PARAMETERS

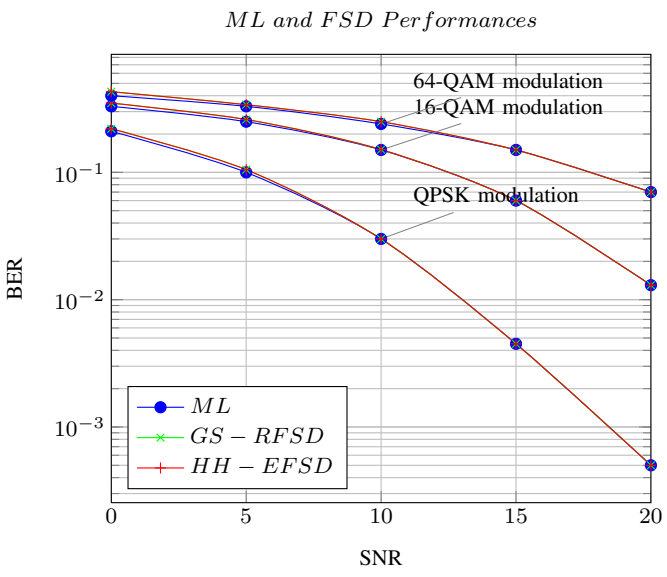

Fig. 6. ML, GS-RFSD and HH-EFSD techniques comparison for QPSK, 16QAM and 64QAM modulations.

ered. Therefore, the obtained performances are exactly the same with GS-RFSD and with HH-EFSD for the announced complexity. Moreover, it is possible to check the HH-EFSD performance is very similar to ML performance. Indeed, BER performances are exactly the same in high SNR case, and a tiny offset is observed in low SNR case which recalls the FSD is suboptimal.

\section{CONCLUSION}

In this paper, the HH-EFSD technique has been presented. The presented technique performance in terms of BER has been shown to be very similar to ML technique and its computational complexity has been shown to be decreased thanks to the HH-based QRD as soon as 5 or more PE are needed. This enhancement during the preprocessing step implies an effective gain of complexity in LTE context in which it has been shown that the mean PE is higher than this announced threshold.

\section{ACKNOWLEDGMENT}

The authors would like to thank Marc Soler and Andrea Ancora for their helpful discussions. This work was supported by ST-NXP Wireless.

\section{REFERENCES}

[1] S. Verdú, Multiuser Detection. Cambridge University Press, 1998.

[2] E. Agrell, T. Eriksson, A. Vardy, and K. Zeger, "Closest point search in lattices," Information Theory, IEEE Transactions on, vol. 48, no. 8, pp. 2201-2214, 2002.

[3] A. Burg, N. Felber, and W. Fichtner, "A $50 \mathrm{mbps} 4 \times 4$ maximum likelihood decoder for multiple-input multiple-output systems with qpsk modulation," in Circuits and Systems, IEEE International Conference on, 2003, pp. 322-325.

[4] W. Mow, "Maximum likelihood sequence estimation from the lattice viewpoint," Information Theory, IEEE Transactions on, vol. 40, no. 5 , pp. 1591-1600, 1994.

[5] U. Fincke and M. Pohst, "Improved methods for calculating vectors of short length in a lattice, including a complexity analysis," Mathematics of Computation, vol. 44, pp. 463-471, 1985.

[6] E. Viterbo and J. Boutros, "A universal lattice code decoder for fading channels," Information Theory, IEEE Transactions on, vol. 45, no. 5, pp. 1639-1642, 1999.

[7] J. Jalden and B. Ottersten, "On the complexity of sphere decoding in digital communications," Signal Processing, IEEE Transactions on, vol. 53, no. 4, pp. 1474-1484, 2005.

[8] L. Barbero and J. Thompson, "Performance analysis of a fixedcomplexity sphere decoder in high-dimensional mimo systems," vol. 4, 2006.

[9] N. Higham, Accuracy and Stability of Numerical Algorithms. Siam, 2002.

[10] L. Barbero and J. Thompson, "Fpga design considerations in the implementation of a fixed-throughput sphere decoder for mimo systems," pp. 1-6, 2006.

[11] B. Hassibi and H. Vikalo, "On the sphere-decoding algorithm i. expected complexity," Signal Processing, IEEE Transactions on, vol. 53, no. 8 Part 1, pp. 2806-2818, 2005.

[12] S. Eckert, "Investigations on different approaches for equalization in mimo ofdm systems in terms of performance and complexity," 2006

[13] Z. Guo and P. Nilsson, "A vlsi architecture of the schnorr-euchner decoder for mimo systems," Circuits and Systems, Symposium on, vol. 1, pp. 65-68, 2004.

[14] A. Chan and A. Lee, "A new reduced-complexity sphere decoder for multiple antenna systems," Communications, IEEE International Conference on, vol. 1, pp. 460-464, 2002.

[15] E.-U. Technical Specification Group RAN, "36.211 physical channels and modulation, v8.0.0," Tech. Rep., 2007. 